\title{
Applying the Wiener-Hermite Random Technique to Study the Evolution of Excess Weight Population in the Region of Valencia (Spain)
}

\author{
J.-C. Cortés", J.-V. Romero, M.-D. Roselló, R.-J. Villanueva \\ Instituto Universitario de Matemática Multidisciplinar, Universitat Politècnica de València, \\ Valencia, Spain \\ Email: *jccortes@imm.upv.es, jvromero@imm.upv.es,drosello@imm.upv.es, rjvillan@imm.upv.es
}

Received July 16, 2012; revised September 20, 2012; accepted October 3, 2012

\begin{abstract}
This paper proposes a stochastic model to study the evolution of normal and excess weight population between 24 - 65 years old in the region of Valencia (Spain). An approximate solution process of the random model is obtained by taking advantage of Wiener-Hermite expansion together with a perturbation method (WHEP). The random model takes as starting point a classical deterministic SIS-type epidemiological model in order to improve it in several ways. Firstly, the stochastic model enhances the deterministic one because it considers uncertainty in its formulation, what it is considered more realistic in dealing with a complex problem as obesity is. Secondly, WHEP approach provides valuable information such as average and variance functions of the approximate solution stochastic process to random model. This fact is remarkable because other techniques only provide predictions in some a priori chosen points. As a consequence, we can compute and predict the expectation and the variance of normal and excess weight population in the region of Valencia for any time. This information is of paramount value to both doctors and health authorities to set optimal investment policies and strategies.
\end{abstract}

Keywords: Random SIS Type—Epidemiological Model; Wiener—Hermite Expansion; Perturbation Method

\section{Introduction}

In the physical, engineering, economical or epidemiological sciences, random differential equations arise in a quite natural manner in the description of models. In fact, numerous phenomena of interest in these areas, which are very important for scientific and technological progress, have been traditionally formulated through mathematical models based on ordinary or partial differential equations, where the data (initial conditions, source term and/or coefficients) are expressed by means of numerical values or deterministic functions. Nevertheless, scientists really set these data from measurements, which always are subject to error. Depending on the quality of these measurements (which frequently can take a lot of time and high cost), the results obtained from the model may be satisfactory. In addition to measurement errors, we should consider the random character of complex external factors that can affect the system, such as pressure, temperature and humidity in Meteorology; the composition of the land in Seismology; investor tendency and economical policy of countries and companies in Finance; the environmental and genetical factors in Epidemiology;

\footnotetext{
${ }^{*}$ Corresponding author.
}

etc. These circumstances make more advisable to consider the data as random magnitudes. The consideration of these facts leads to the reformulation of the traditional deterministic models, which, in order to improve them, should be replaced by random models.

However, even recognizing the necessity of considering a random approach in the formulation of such models, often it is very difficult, if not impossible, to establish a suitable way to model uncertainty due to the complexity involved in the specific problem under study. Based on the central limit theorem, a popular and successful way to model randomness is through a Gaussian process having additional mathematical properties such as white noise does. White noise is a stationary Gaussian stochastic process with mean value zero and a constant spectral density on the entire real axis. Interesting contributions in the modeling of applied problems in different fields using white noise can be found in [1-6], for instance.

In this paper we are interested in forecasting the evolution of excess and normal weight population in the region of Valencia (Spain) by means of a random model that involves white noise. Due to the lack of statistical data about the problem under study, this stochastic model 
takes advantage of certain information obtained from a deterministic SIS-type epidemic model. In fact, as we will see, the random model takes as starting point useful conclusions provided by the classical deterministic approach. In this way, the random model improves the deterministic one because it considers uncertainty in its formulation, what it is considered more realistic. In addition, this approach provides valuable information such as average and variance functions of the approximate solution stochastic process.

As it is shown in Section 2, this epidemiological model can be described as a particular case of the following random differential equation

$$
\begin{aligned}
& \dot{x}(t)=a(t)+b(t) x(t)+\varepsilon(x(t))^{2}+\lambda n(t), \\
& t>0, \quad x(0)=x_{0},
\end{aligned}
$$

where coefficients $a(t), b(t)$ and initial condition $x_{0}$ are deterministic, $\varepsilon$ is a small parameter and

$n(t)=n(t)(\omega)$ is a white noise process, which intensity is modulated by parameter $\lambda$. By $\omega$, we denote a random outcome of a probability space $(\Omega, \mathcal{A}, P)$, where $\Omega$ is a sample space, $\mathcal{A}$ is a $\sigma$-algebra associate to $\Omega$ and, $P$ is a probability measure.

As we will see throughout the subsequent development, the method used to obtain the approximate solution to Equation (1) is based on the so-called Wiener-Hermite expansion (WHE). WHE constitutes a powerful technique to represent any stochastic process in terms of the so-called Wiener-Hermite polynomials as well as certain deterministic kernels to be calculated. Interesting contributions where this technique have been used successfully to solve other class of random differential equations can be found in references [7-10] and other contained therein.

The article is organized as follows. In Section 2 we establish a random model of type (1) in order to study excess weight population aged between 24 - 65 years old in the region of Valencia (Spain). The stochastic model arises in a natural way by introducing uncertainty in the corresponding deterministic SIS-type epidemiological model. In Section 3 we first summarize the main results about the WHE method and then, we apply it to derive a coupled integro-differential system that is satisfied by the involved kernels. This section concludes with the application of the perturbation technique to conduct the resolution of such a system. Section 4 is devoted to solve the random SIS-type epidemiological model presented in Section 2 by taking advantage of development given in Section 3. Conclusions are discussed in Section 5.

\section{Motivating the Mathematical Model}

Some mathematical models to deal with the evolution over time of excess weight populations have been recently developed [11,12]. In [12] it is presented a deter- ministic differential mathematical model to predict the future evolution of the 3 - 5 years old infant excess weight population in the region of Valencia (Spain) over a finite time. In [11] the study is developed for the whole population and an asymptotic behavior analysis is presented. Both papers consider obesity as a health concern that spreads by social peer pressure and social contact through unhealthy lifestyle habits [11-13]. These contributions are based on epidemiological models [14]. Although more complex deterministic models to study excess weight population have been proposed $[12,15,16]$, in this paper we want, in a first step, to explore by means of a simple but representative type-model, the ability of Wiener-Hermite expansion to provide a suitable approach to deal with such a class of models that include uncertainty in their formulation. It could permit the extension to this approach more sophisticated models in future works.

In order to motivate the statement of the random model, we first take the corresponding SIS-epidemiological deterministic one as starting point. Hereinafter, we concentrate on population aged between 24 - 65 in the region of Valencia (Spain). In this study, we consider that population is partitioned into two subpopulations, $N(t)$ and $O(t)$, that denote the proportion of normal and excess weight individuals at time $t$, respectively. Without loss of generality, we assume that the whole population is normalized to unit, i.e., $N(t)+O(t)=1$ for all time $t$. Following an analogous reasoning as it is given in $[11,12]$, the model can be represented by the following two-state dynamical coupled nonlinear system:

$$
\left.\begin{array}{l}
N^{\prime}(t)=\mu N_{0}-\mu N(t)-\beta N(t) O(t)+\rho O(t), \\
O^{\prime}(t)=\mu O_{0}+\beta N(t) O(t)-(\mu+\rho) O(t),
\end{array}\right\}
$$

with initial conditions $N(0)$ and $O(0)$. Time invariant parameters for system (2.1) are:

- $\mu$, average stay time in the system of $24-65$ years old adults.

- $\quad \rho$, rate at which an excess weight individual moves to normal weight subpopulation.

- $\quad \beta$, transmission rate due to social pressure to adopt an unhealthy lifestyle (TV, friends, family, job).

- $N_{0}$, proportion of normal weight population coming from the 23 years old age group.

- $\mathrm{O}_{0}$, proportion of excess weight population coming from the 23 years old age group.

System (2.1) can be interpreted as a SIS-type compartmental model which dynamic of transits between subpopulations is depicted in Figure 1.

Since $N(t)+O(t)=1$, system (2.1) can be simplified to only one nonlinear differential equation involving as unique unknown the percentage of normal weight people 


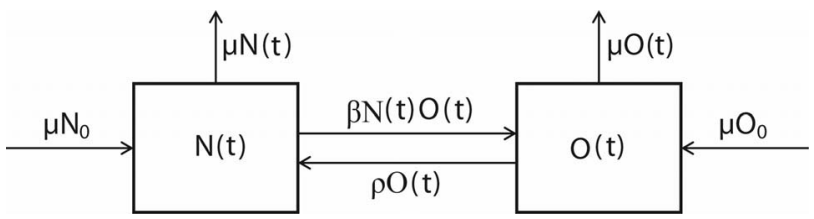

Figure 1. Flow diagram of the deterministic model for the dynamic of obesity prevalence in the population.

$$
N^{\prime}(t)=A+B N(t)+C(N(t))^{2},
$$

for a given initial condition $N(0)$, being $A=\mu N_{0}+\rho$, $B=-(\mu+\rho+\beta), C=\beta$.

Following an analogous methodology as in $[12,15,16]$, parameter $\beta$ is estimated by fitting the model with data from the Health Survey of the Region of Valencia 2000 and $2005[17,18]$. The other parameters are estimated using the same Health Survey and [19]. Table 1 collects these values where time variable $t$ is measured in weeks.

However, note that the aforementioned deterministic model does not take into account nor the inherent errors in the measured data provided by the Health Survey neither inherent complexity of obesity such as individual behavior, geographical conditions, genetic aspects, health advertising campaigns, etc. When data are available to inform us about the best choice for data distribution, the parameter assignment is easily made. However, in the lack of data of this sort of information on the distribution for a specific parameter or, even more, for the randomness affecting a complex problem as obesity is, the specification of such information is very difficult, if not impossible, to get. White noise stochastic process has demonstrated to be a powerful tool to model properly general uncertainty [20,21]. In this paper, based on this consideration, we propose to modify the obesity model (2.1), considering that the dynamic of normal weight subpopulation is described by the random differential equation:

$$
N^{\prime}(t)=A+B N(t)+C(N(t))^{2}+\lambda n(t),
$$

where $n(t)=n(t)(\omega)$ is a white noise process, which intensity is given by parameter $\lambda$. This model is just a particular case of (1), where coefficients are now assumed to be time-independent. Notice that in the real problem we are interested to apply the random model, from Table 1, it is plausible to assume that $C=\beta$ is a small parameter.

The previous exposition leads us to face several new problems that need to be answered. Firstly, we now have to solve the random differential Equation (2.3) or the more general, (1). Secondly, taking the deterministic model as a starting point, we have to fit parameter $\lambda$ in order to provide a complete description of obesity model through random approach. This motivates the next section which is devoted to obtain an approximate solution
Table 1. Initial conditions and parameter values for the SIS model (2.1).

\begin{tabular}{ccccccc}
\hline$N(0)$ & $O(0)$ & $\beta$ & $\rho$ & $\mu$ & $N_{0}$ & $O_{0}$ \\
\hline 0.522 & 0.488 & 0.00085 & 0.000035 & 0.000469 & 0.704 & 0.296 \\
\hline
\end{tabular}

stochastic process to random differential Equation (1). In Section 4, we apply these results to specify an approximate solution of obesity model.

\section{Applying the Wiener-Hermite Expansion (WHE) Together with the Perturbation Method to Approximate the General Nonlinear Stochastic Solution}

To approximate the solution stochastic process of model (1), a truncation of the Wiener-Hermite expansion (WHE) together with the perturbation method is employed. This technique, denoted by WHEP, was firstly introduced in [22]. WHE is based on the Wiener-Hermite (WH) polynomials. These polynomials constitute a complete set of statistically orthogonal random processes which was introduced in [23] (see also [24]). The first few terms of the Wiener-Hermite polynomials are:

$$
\begin{aligned}
& H^{(0)}=1, \\
& H^{(1)}(t)=n(t), \\
& H^{(2)}\left(t_{1}, t_{2}\right)=n\left(t_{1}\right) n\left(t_{2}\right)-\delta\left(t_{1}-t_{2}\right), \\
& H^{(3)}\left(t_{1}, t_{2}, t_{3}\right)=n\left(t_{1}\right) n\left(t_{2}\right) n\left(t_{3}\right)-n\left(t_{1}\right) \delta\left(t_{2}-t_{3}\right) \\
& -n\left(t_{2}\right) \delta\left(\left(t_{1}-t_{3}\right)-n\left(t_{3}\right) \delta\left(t_{1}-t_{2}\right),\right.
\end{aligned}
$$

where $n(t)$ denotes a white noise process which, by definition, is centered at the origin and its correlation is the Dirac delta function:

$$
\mathrm{E}[n(t)]=0, \quad \mathrm{E}\left[n\left(t_{1}\right) n\left(t_{2}\right)\right]=\delta\left(t_{1}-t_{2}\right) .
$$

More generally, taking $H^{(0)}$ and $H^{(1)}(t)$ as starting values, WH polynomials, denoted by $H^{(i)}=H^{(i)}\left(t_{1}, \cdots, t_{i}\right)$, can be defined recurrently as follows:

$$
\begin{aligned}
& H^{(i)}\left(t_{1}, \cdots, t_{i}\right) \\
& =H^{(i-1)}\left(t_{1}, \cdots, t_{i-1}\right) H^{(1)}\left(t_{i}\right) \\
& -\sum_{j=1}^{i-1} H^{(i-2)}\left(t_{i_{1}}, \cdots, t_{i_{i-2}}\right) \delta\left(t_{i-j}-t_{i}\right), \quad i \geq 2,
\end{aligned}
$$

where for each $i \geq 2$ and $j \in\{1, \cdots, i-1\}$, subindex set $\left\{i_{1}, \cdots, i_{i-2}\right\}$ is extracted from the original subindex set $\{1,2, \cdots, i\}$ keeping their order and excluding numbers $i-j$ and $i$.

Taking advantage of property (3.1), one can demonstrate that $\mathrm{WH}$ polynomials satisfy:

$$
\mathrm{E}\left[H^{(0)}\right]=1, \quad \mathrm{E}\left[H^{(i)}\right]=0, \forall i \geq 1,
$$


as well as they are statistically orthogonal:

$$
\mathrm{E}\left[H^{(i)} H^{(j)}\right]=0, \forall i \neq j .
$$

As a consequence of the completeness of the WH set [23,24], any arbitrary stochastic process, say $x(t)=x(t ; \omega), \omega \in \Omega$, can be expanded in terms of a $\mathrm{WH}$ polynomials set and this expansion converges to the original stochastic process, i.e.,

$$
\begin{aligned}
& x(t)=x^{(0)}(t)+\int_{\mathbb{R}} x^{(1)}\left(t ; t_{1}\right) H^{(1)}\left(t_{1}\right) \mathrm{d} t_{1} \\
& +\int_{\mathbb{R}^{2}} x^{(2)}\left(t ; t_{1}, t_{2}\right) H^{(2)}\left(t_{1}, t_{2}\right) \mathrm{d} t_{1} \mathrm{~d} t_{2}+\cdots,
\end{aligned}
$$

where $x^{(0)}=x^{(0)}(t), \quad x^{(i)}=x^{(i)}\left(t ; t_{1}, \cdots, t_{i}\right), \quad i \geq 1$ are called the (deterministic) kernels of the WHE of $x(t)$. The first two terms of the right-hand side define the Gaussian representation of $x(t)$ (being the zeroth-order term just its mean or average, i.e., $\left.\mathrm{E}[x(t)]=x^{(0)}(t)\right)$, while the second and higher-order terms correspond to the non-Gaussian part. The variance of $x(t)$ can be expressed as follows:

$$
\begin{aligned}
\operatorname{Var}[x(t)] & =\int_{\mathbb{R}}\left(x^{(1)}\left(t ; t_{1}\right)\right)^{2} \mathrm{~d} t_{1} \\
& +2 \int_{\mathbb{R}^{2}}\left(x^{(2)}\left(t ; t_{1}, t_{2}\right)\right)^{2} \mathrm{~d} t_{1} \mathrm{~d} t_{2}+\cdots .
\end{aligned}
$$

The basic method to obtain an approximate solution stochastic process of model (1) by taking advantage of WHE technique is, in a first step, to consider a truncation of the infinite expansion (3.4) for $x(t)$. Let $N$ be the order of such a truncation. This entails that we need to compute $N+1$ deterministic kernels $x^{(i)}, i=0,1, \cdots, N$ to get an approximation based on (3.4). This can be made by deriving $N+1$ integro-differential equations for the dynamics of the unknown kernel functions. This set of deterministic equations is established by taking advantage of the stochastic orthogonality properties of $\mathrm{WH}$ polynomials. Henceforth, we take $N=2$, so we are going to compute the first non-Gaussian approximation of $x(t)$. Therefore, to be precise, three integro-differential equations for $x^{(0)}(t), x^{(1)}\left(t ; t_{1}\right)$ and $x^{(2)}\left(t ; t_{1}, t_{2}\right)$ need to be established.

In order to derive the first equation, we just follow previous procedure: we substitute the corresponding truncated WHE of $x(t)$ obtained from (3.4) in the model (1). Next we take the expectation operator over the resulting expression and then we apply properties (3.1)-(3.3) together with

$$
\begin{gathered}
\mathrm{E}\left[H^{(1)}\left(t_{1}\right) H^{(1)}\left(t_{2}\right)\right]=\delta\left(t_{1}-t_{2}\right), \\
\mathrm{E}\left[H^{(2)}\left(t_{1}, t_{2}\right) H^{(2)}\left(t_{3}, t_{4}\right)\right]=\delta\left(t_{1}-t_{3}\right) \delta\left(t_{2}-t_{4}\right) \\
+\delta\left(t_{1}-t_{4}\right) \delta\left(t_{2}-t_{3}\right) .
\end{gathered}
$$

This leads to

$$
\left.\begin{array}{rl}
\dot{x}^{(0)}(t)= & a(t)+b(t) x^{(0)}(t) \\
+ & \varepsilon\left\{\left(x^{(0)}(t)\right)^{2}+\int_{\mathbb{R}}\left(x^{(1)}\left(t ; t_{1}\right)\right)^{2} \mathrm{~d} t_{1}\right. \\
& \left.+2 \int_{\mathbb{R}^{2}}\left(x^{(2)}\left(t ; t_{1}, t_{2}\right)\right)^{2} \mathrm{~d} t_{1} \mathrm{~d} t_{2}\right\}
\end{array}\right\}
$$

The initial condition has been derived by setting $t=0$ in (3.4), then applying the expectation operator and finally taking advantage of property (3.2).

Now, we address to establish a second (deterministic) differential equation for $x^{(1)}\left(t ; t_{1}\right)$. For that, we firstly multiply the corresponding truncated WHE (3.4) of $x(t)$ by $H^{(1)}\left(t_{5}\right)$. Then we take the expectation operator and, we again apply above properties together with

$$
\begin{aligned}
& \mathrm{E}\left[H^{(1)}\left(t_{1}\right) H^{(1)}\left(t_{2}\right) H^{(1)}\left(t_{3}\right)\right] \\
& =\mathrm{E}\left[H^{(1)}\left(t_{1}\right) H^{(2)}\left(t_{2}, t_{3}\right) H^{(2)}\left(t_{4}, t_{5}\right)\right]=0, \\
& \mathrm{E}\left[H^{(1)}\left(t_{1}\right) H^{(1)}\left(t_{2}\right) H^{(2)}\left(t_{3}, t_{4}\right)\right] \\
& =\delta\left(t_{1}-t_{3}\right) \delta\left(t_{2}-t_{4}\right)+\delta\left(t_{1}-t_{4}\right) \delta\left(t_{2}-t_{3}\right) .
\end{aligned}
$$

In this case, one gets

$$
\begin{aligned}
& \dot{x}^{(1)}\left(t ; t_{1}\right)=b(t) x^{(1)}\left(t ; t_{1}\right)+\lambda \delta\left(t-t_{1}\right) \\
& +\varepsilon\left\{2 x^{(0)}(t) x^{(1)}\left(t ; t_{1}\right)+4 \int_{\mathbb{R}} x^{(1)}\left(t ; t_{2}\right) x^{(2)}\left(t ; t_{1}, t_{2}\right) \mathrm{d} t_{2}\right\}, \\
& x^{(1)}\left(0 ; t_{1}\right)=0, \forall t_{1} .
\end{aligned}
$$

In this case, the initial condition has been established multiplying by $H^{(1)}\left(t_{3}\right)$ the truncated WHE (3.4), then we set $t=0$ and take the expectation operator, and finally, we apply properties (3.2) and (3.6).

The initial value problem that has been established in order to compute the kernel $x^{(2)}\left(t ; t_{1}, t_{2}\right)$ is given by

$$
\left.\begin{array}{rl}
\dot{x}^{(2)}\left(t ; t_{1}, t_{2}\right)= & b(t) x^{(2)}\left(t ; t_{1}, t_{2}\right) \\
+ & \varepsilon\left\{x^{(1)}\left(t ; t_{1}\right) x^{(1)}\left(t ; t_{2}\right)+2 x^{(0)}(t) x^{(2)}\left(t ; t_{1}, t_{2}\right)\right. \\
& \left.+4 \int_{\mathbb{R}} x^{(2)}\left(t ; t_{3}, t_{1}\right) x^{(2)}\left(t ; t_{3}, t_{2}\right) \mathrm{d} t_{3}\right\}
\end{array}\right\}
$$

To obtain this equation, firstly we have multiplied the corresponding truncated WHE (3.4) of $x(t)$ by $H^{(2)}\left(t_{5}, t_{6}\right)$. Next we have taken the expectation operator and then we have applied previous properties together with 


$$
\begin{aligned}
& \mathrm{E}\left[H^{(2)}\left(t_{1}, t_{2}\right) H^{(2)}\left(t_{3}, t_{4}\right) H^{(2)}\left(t_{5}, t_{6}\right)\right] \\
& =\delta\left(t_{1}-t_{3}\right) \delta\left(t_{2}-t_{5}\right) \delta\left(t_{4}-t_{6}\right) \\
& +\delta\left(t_{1}-t_{3}\right) \delta\left(t_{2}-t_{6}\right) \delta\left(t_{4}-t_{5}\right) \\
& +\delta\left(t_{1}-t_{4}\right) \delta\left(t_{2}-t_{5}\right) \delta\left(t_{3}-t_{6}\right) \\
& +\delta\left(t_{1}-t_{4}\right) \delta\left(t_{2}-t_{6}\right) \delta\left(t_{3}-t_{5}\right) \\
& +\delta\left(t_{1}-t_{5}\right) \delta\left(t_{2}-t_{3}\right) \delta\left(t_{4}-t_{6}\right) \\
& +\delta\left(t_{1}-t_{5}\right) \delta\left(t_{2}-t_{4}\right) \delta\left(t_{3}-t_{6}\right) \\
& +\delta\left(t_{1}-t_{6}\right) \delta\left(t_{2}-t_{3}\right) \delta\left(t_{4}-t_{5}\right) \\
& +\delta\left(t_{1}-t_{6}\right) \delta\left(t_{2}-t_{4}\right) \delta\left(t_{3}-t_{5}\right) .
\end{aligned}
$$

The initial condition has been established multiplying by $H^{(2)}\left(t_{3}, t_{4}\right)$ the truncated WHE (3.4), then we set $t=0$ and take the expectation operator, and finally, we apply properties (3.2) and (3.7).

As $\varepsilon>0$ is assumed to be a frank small parameter, a reliable technique to solve nonlinear coupled deterministic problems (3.8)-(3.10) is the perturbation method [22]. We represent the deterministic kernels by means of their first approximations as follows:

$$
\begin{aligned}
& x^{(0)}(t)=x_{0}^{(0)}(t)+\varepsilon x_{1}^{(0)}(t), \\
& x^{(1)}\left(t ; t_{1}\right)=x_{0}^{(1)}\left(t ; t_{1}\right)+\varepsilon x_{1}^{(1)}\left(t ; t_{1}\right) \\
& x^{(2)}\left(t ; t_{1}, t_{2}\right)=x_{0}^{(2)}\left(t ; t_{1}, t_{2}\right)+\varepsilon x_{1}^{(2)}\left(t ; t_{1}, t_{2}\right)
\end{aligned}
$$

Now, we substitute these representations in Equations (3.8)-(3.10) and we neglect those powers of $\varepsilon$ which exponents are greater than 1 . Hence we obtain the follow-ing initial value problems:

$$
\begin{gathered}
\left.\begin{array}{c}
\dot{x}_{0}^{(0)}(t)=a(t)+b(t) x_{0}^{(0)}(t), \\
x_{0}^{(0)}(0)=x_{0},
\end{array}\right\} \\
\dot{x}_{1}^{(0)}(t)=b(t) x_{1}^{(0)}(t)+\left(x_{0}^{(0)}(t)\right)^{2}+\int_{0}^{\infty}\left(x_{0}^{(1)}\left(t ; t_{1}\right)\right)^{2} \mathrm{~d} t_{1} \\
+2 \int_{0}^{\infty} \int_{0}^{\infty}\left(x_{0}^{(2)}\left(t ; t_{1}, t_{2}\right)\right)^{2} \mathrm{~d} t_{1} \mathrm{~d} t_{2}, \\
\left.\begin{array}{rl}
x_{1}^{(0)}(0)=0, \\
\dot{x}_{0}^{(1)}\left(t ; t_{1}\right)=b(t) x_{0}^{(1)}\left(t ; t_{1}\right)+\lambda \delta\left(t-t_{1}\right), \\
x_{0}^{(1)}\left(0 ; t_{1}\right)=0, \forall t_{1} \geq 0 \\
\dot{x}_{1}^{(1)}\left(t ; t_{1}\right)=b(t) x_{1}^{(1)}\left(t ; t_{1}\right)+2 x_{0}^{(0)}(t) x_{0}^{(1)}\left(t ; t_{1}\right) \\
+4 \int_{0}^{\infty} x_{0}^{(1)}\left(t ; t_{2}\right) x_{0}^{(2)}\left(t ; t_{1}, t_{2}\right) \mathrm{d} t_{2}, \\
x_{1}^{(1)}\left(0 ; t_{1}\right)=0, \forall t_{1} \geq 0
\end{array}\right\}
\end{gathered}
$$

From these expressions, we then obtain the solution of (3.12), (3.14) and (3.16), although due to its cumbersome representation we do not explicit $x_{1}^{(2)}(t)$ here.

$$
\begin{aligned}
& x_{1}^{(0)}(t)=-\frac{1}{2 b^{3}}\left\{-2 a^{2}+b \lambda^{2}+\mathrm{e}^{2 b t}\left[b \lambda^{2}+2\left(a+b x_{0}\right)^{2}\right]\right. \\
& \left.\quad-2 b \mathrm{e}^{b t}\left[\lambda^{2}+2 a^{2} t+b\left(x_{0}\right)^{2}+2 a x_{0}(1+b t)\right]\right\}, \\
& x_{1}^{(1)}\left(t ; t_{1}\right) \\
& =\left\{\begin{array}{cc}
-\frac{2 \lambda}{b^{2}} \mathrm{e}^{b\left(t-t_{1}\right)}\left[\left(\mathrm{e}^{b t_{1}}-\mathrm{e}^{b t}\right)\left(a+b x_{0}\right)+a b\left(t-t_{1}\right)\right] \\
\text { if } t \geq t_{1}, & \text { if } t<t_{1},
\end{array}\right.
\end{aligned}
$$

Taking into account that $\mathrm{E}[x(t)]=x^{(0)}(t) \approx x_{0}^{(0)}(t)+\varepsilon x_{1}^{(0)}(t)$, one gets the following approximation of the mean of $x(t)$ which depends on parameters $a, b, \lambda, x_{0}$ :

\section{Solving the Random SIS-Type}

In this section we focus on the solution of random SIStype epidemiological model given by (2.3) which is a particular case of (1.1). Therefore, hereinafter we will pers we can obtain directly the solution of initial value problems (3.11)-(3.16). In fact, we firstly compute the solution to (3.11), (3.13) and (3.15):

$$
\begin{aligned}
& x_{0}^{(0)}(t)=\left(x_{0}+\frac{a}{b}\right) \mathrm{e}^{b t}-\frac{a}{b}, \\
& x_{0}^{(1)}\left(t ; t_{1}\right)=\left\{\begin{array}{cc}
\lambda \mathrm{e}^{b\left(t-t_{1}\right)} & \text { if } t \geq t_{1}, \\
0 & \text { if } t<t_{1},
\end{array}\right. \\
& x_{0}^{(2)}\left(t ; t_{1}, t_{2}\right)=0, \forall t_{1}, t_{2} \geq 0 .
\end{aligned}
$$




$$
\begin{aligned}
& \mathrm{E}\left[x\left(t ; a, b, \lambda, x_{0}\right)\right] \approx\left(x_{0}+\frac{a}{b}\right) \mathrm{e}^{b t}-\frac{a}{b} \\
& -\varepsilon\left\{\frac { 1 } { 2 b ^ { 3 } } \left\{-2 a^{2}+b \lambda^{2}+\mathrm{e}^{2 b t}\left[b \lambda^{2}+2\left(a+b x_{0}\right)^{2}\right]\right.\right. \\
& \left.\left.-2 b \mathrm{e}^{b t}\left[\lambda^{2}+2 a^{2} t+b\left(x_{0}\right)^{2}+2 a x_{0}(1+b t)\right]\right\}\right\}
\end{aligned}
$$

In order to compute an approximation of the expectation function of the solution stochastic process to SIStype epidemiological model, we identify coefficients: $a=A=\mu N_{0}+\rho=0.000365176$,

$b=B=-(\mu+\rho+\beta)=-0.001354$, $\varepsilon=C=\beta=0.00085$ and the initial condition $x_{0}=N(0)$. These numerical values have been computed from Table 1. Note that in the context of our problem, the only available data are $N(0)=0.522$ and $N(260)=0.49$, that correspond to the percentage of normal weight people aged between 24 - 65 in the region of Valencia (Spain). These percentages have been obtained from the Health Surveys of the Region of Valencia of the years 2000 and 2005, respectively. Note that time variable $t$ is measured in weeks. While

$N(0)=0.522$ has been used as initial condition, we can take advantage of information in $t=260$ together with (4.1) to fit the intensity $\lambda$ of the white noise. Indeed, we impose that the only available value of the stochastic process $N(t)=N(t)(\omega)$ representing the percentage of normal weight population at $t=260$ coincides with the (approximate) expectation:

$$
\mathrm{E}[x(260 ; 0.000365176,-0.001354, \lambda, 0.522)]=0.49,
$$

which solution is $\lambda=0.0155689$. From expression (4.1), we can now obtain a full approximation of the expectation function of the percentage of the normal weight population. On the left side of Figure 2, we represent this approximation over the weekly interval $[0,780]$ that corresponds to the yearly interval [2000, 2015].

Notice the proposed method leads to an approximation of the expectation that is also a function (see (4.1)) and this point constitutes one of the most relevant contribution of the mixed random-deterministic model. In fact, alternative approaches based on the deterministic model (2.1) or equivalently (2.2) using Monte Carlo methods as Latin Hypercube Sampling [25] only provide predictions in specific time points, while using (4.1), we can obtain an approximation to the expectation in any time.

To complete the stochastic approach, we now address the computation of an approximation of the variance function. Since $x_{0}^{(2)}\left(t ; t_{1}, t_{2}\right)=0$, by perturbation method and (3.5), neglecting terms of $\varepsilon$ with power greater than 1 , one gets

$$
\operatorname{Var}[x(t)] \approx \int_{-\infty}^{\infty}\left(\left(x_{0}^{(1)}\left(t ; t_{1}\right)\right)^{2}+2 \varepsilon x_{0}^{(1)}\left(t ; t_{1}\right) x_{1}^{(1)}\left(t ; t_{1}\right)\right)^{2} \mathrm{~d} t_{1} \text {. }
$$
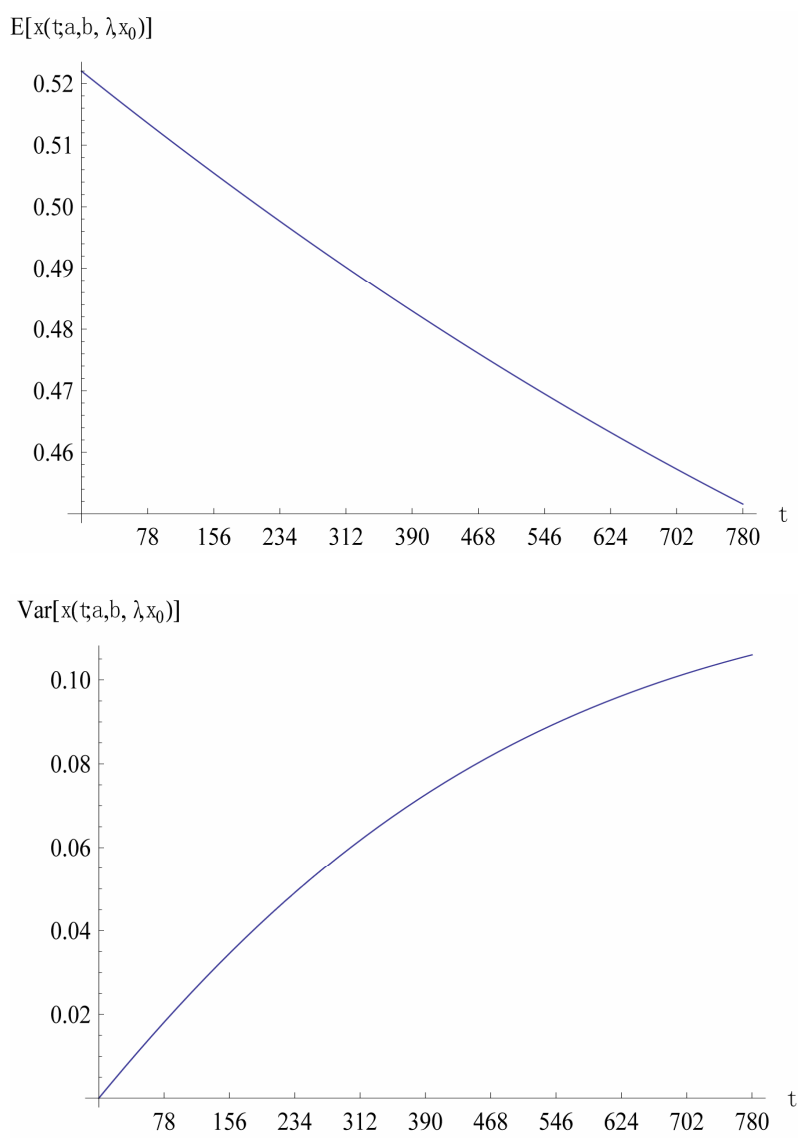

Figure 2. Wiener-Hermite approximation of the expectation (left) and variance (right) of the normal weight population model (1) with $a=0.000365176, b=-0.001354, \lambda=$ 0.0155689 and $x_{0}=0.522$ between years 2000 and 2015 (corresponding to the interval $[0,780]$ ).

On the right side of Figure 2, we plot

$\operatorname{Var}[x(t)]=\operatorname{Var}\left[x\left(t ; a, b, \lambda, x_{0}\right)\right]$. Although from a practical view-point is not realistic to enlarge the time interval beyond 2015 (that corresponds to 780 weeks), we have checked that expectation stabilizes over time, and as a consequence the variance does.

\section{Conclusion}

In this paper a Wiener-Hermite random technique together with a perturbation method has been developed and applied to a two-state dynamical system to study the evolution (transmission dynamics) of excess and normal weight of adults between 24 - 65 years old in the Spanish region of Valencia, and we have obtained an approximation of the expectation and variance functions. Both approximations are also functions and this fact is remarkable because other techniques only provides predictions in some a priori chosen time-points. As a consequence, we can compute and predict the expectation and the variance of normal weight population in the region of Valencia for 
any time point. We point out that the main aim of this paper is to show the promising potentiality of mixed Wiener-Hermite and perturbation methods to deal with models based on stochastic differential Equation (2.3). Once more data will be available a study of the error would be advisable including the consideration of different number of terms when applying perturbation technique. Our approach reveals, as other sources do, that normal weight population is decreasing gradually, and it is a health concern worrying doctors and local government. Finally, this paper is an example about how models can be a useful tools to experiment with health concerns. Using these type of approaches, health policy members are able to simulate different scenarios and analyze the effect of the change in health policies.

\section{Acknowledgements}

This work has been partially supported by the Spanish M.C.Y.T. and FEDER grants MTM2009-08587, DPI201020891-C02-01 as well as Universidad Politécnica de Valencia grant PAID06-11-2070.

\section{REFERENCES}

[1] M. Joelson and A. Ramamonjiarisoa, "Random Fields of Water Surface Waves Using Wiener-Hermite Functional Series Expansions," Journal of Fluid of Mechanics, Vol. 496, 2003, pp. 313-334. doi:10.1017/S002211200300644X

[2] Y. Kayanuma and D. Nelson, "Wiener-Hermite Expansion Formalism for the Stochastic Model of a Driven Quantum System,” Chemical Physics, Vol. 268, No. 1-3, 2001, pp. 177-188. doi:10.1016/S0301-0104(01)00305-6

[3] F. Black and M. Scholes, "The Pricing of Options and Corporate Liabilities," Journal of Political Economy, Vol. 81, No. 3, 1973, pp. 637-654. doi:10.1086/260062

[4] C. Braumann, "Variable Effort Harvesting Models in Random Environments: Generalization to Density-Dependent Noise Intensities," Mathematical Biosciences, Vol. 177-178, 2002, pp. 229-245. doi:10.1016/S0025-5564(01)00110-9

[5] E. A. Gawad, M. A. El-Tawil and M. A. Nassar, "Nonlinear Oscillatory Systems with Random Excitation,” Modelling, Simulation \& Control B, Vol. 23, No. 1, 1989, pp. 55-63.

[6] M. A. El-Tawil and G. Mahmoud, "The Solvability of Parametrically Forced Oscillators Using WHEP Technique," Mechanics Mechanical Engineering, Vol. 3, No. 2, 1999, pp. 181-188.

[7] M. A. El-Tawil and N. A. Al-Mulla, "Using Homotopy WHEP Technique for Solving a Stochastic Nonlinear Diffusion Equation,” Mathematical and Computer Modelling, Vol. 51, No. 9-10, 2010, pp. 1277-1284. doi:10.1016/j.mcm.2010.01.013

[8] M. A. El-Tawil and N. A. Al-Mulla, "Solving Nonlinear Diffusion Equations without Stochastic Homogeneity
Using Homotopy Perturbation Method," International Journal of Nonlinear Sciences and Numerical Simulation, Vol. 10, No. 5, 2009, pp. 687-698.

[9] M. A. El-Tawil and A. S. Al-Johani, “Approximate Solution of a Mixed Nonlinear Stochastic Oscillator," Computers \& Mathematics with Applications, Vol. 58, No. 11-12, 2009, pp. 2236-2259. doi:10.1016/j.camwa.2009.03.057

[10] A. S. Al-Jihany, "Comparisons between WHEP and Homotopy Perturbation Techniques in Solving Stochastic Cubic Oscillatory Problems," The Open Applied Mathematics Journal, Vol. 4, 2010, pp. 24-30.

[11] A. M. Evangelista, A. R. Ortiz, K. R. Rios-Soto and A. Urdapilleta. "USA the Fast Food Nation: Obesity as an Epidemic.” T-7, MS B284, Theoretical Division, Los Alamos National Laboratory, Los Alamos, NM 87545, 2004.

http://math.lanl.gov/SummerPrograms/Reports2004/ev_o r_ri_ur.pdf

[12] L. Jódar, F. Santonja and G. González-Parra, "Modeling Dynamics of Infant Obesity in the Region of Valencia, Spain," Computers \& Mathematics with Applications, Vol. 56, No. 3, 2008, pp. 679-689. doi:10.1016/j.camwa.2008.01.011

[13] N. A. Christakis and J. H. Fowler, "The Spread of Obesity in a Large Social Network over 32 Years,” The New England Journal of Medicine, Vol. 357, No. 4, 2007, pp. 370-379. doi:10.1056/NEJMsa066082

[14] J. D. Murray, "Mathematical Biology,” Springer, Berlin, 2002.

[15] G. González-Parra, L. Jódar, F. Santonja and R. J. Villanueva, "Age-Structured Model for Childhood Obesity,” Mathematical Population Studies, Vol. 171, No. 1, 2010, pp. 1-17. doi:10.1080/07481180903467218

[16] F. Santonja, R. J. Villanueva, L. Jódar and G. GonzálezParra, "Mathematical Modeling of Social Obesity Epidemic in the Region of Valencia, Spain," Mathematical and Computer Modelling of Dynamical Systems, Vol. 16, No. 1, 2010, pp. 23-34. doi:10.1080/13873951003590149

[17] Valencian Department of Health, "Health Survey, Year 2000," 2010. http://www.san.gva.es/val/prof/homeprof.html

[18] Valencian Department of Health, "Health Survey, Year 2005, 2010. http://www.san.gva.es/val/prof/homeprof.html

[19] J. J. Arrizabalaga, L. Masmiquel, J. Vidal, A. Calaas, M. J. Díaz, P. P. García, S. Monereo, J. Moreiro, B. Moreno, W. Ricart and F. Cordido, "Recomendaciones y Algoritmo de Tratamiento Del Sobrepeso y la Obesidad en Personas Adultas (in Spanish)," Medicina Clínica, Vol. 122, No. 3, 2004, pp. 104-110. doi:10.1157/13056816

[20] E. Kloeden and E. Platen, "Numerical Solution of Stochastic Differential Equations,” Springer, Berlin, 1992.

[21] A. J. Arenas, G. González-Parra and J. A. Moraño, "Stochastic Modelling of the Transmission of Respiratory Synctytial Virus (RSV) in the Region of Valencia (Spain),” Byosystems, Vol. 96, No. 3, 2009, pp. 206-212. doi:10.1016/j.biosystems.2009.01.007 
[22] E. A. Gawad and M. A. El-Tawil, "General Stochastic Oscillatory Systems,” Applied Mathematical Modelling, Vol. 17, No. 6, 1993, pp. 329-335. doi:10.1016/0307-904X(93)90058-O

[23] N. Wiener, "Nonlinear Problems in Random Theory," MIT Press, New York, 1958.

[24] R. H. Cameron and W. T. Martin, "The Orthogonal De- velopment of Non-Linear Functionals in Series of Fourier-Hermite Functionals," Annals of Mathematics, Vol. 48, No. 2, 1947, pp. 385-392. doi:10.2307/1969178

[25] S. Marino, I. B. Hogue, C. J. Ray and D. E. Kirschner, “A Methodology for Performing Global Uncertainty and Sensititivity Analysis in Systems Biology," Journal of Theoretical Biology, Vol. 254, 2008, pp. 178-196. 